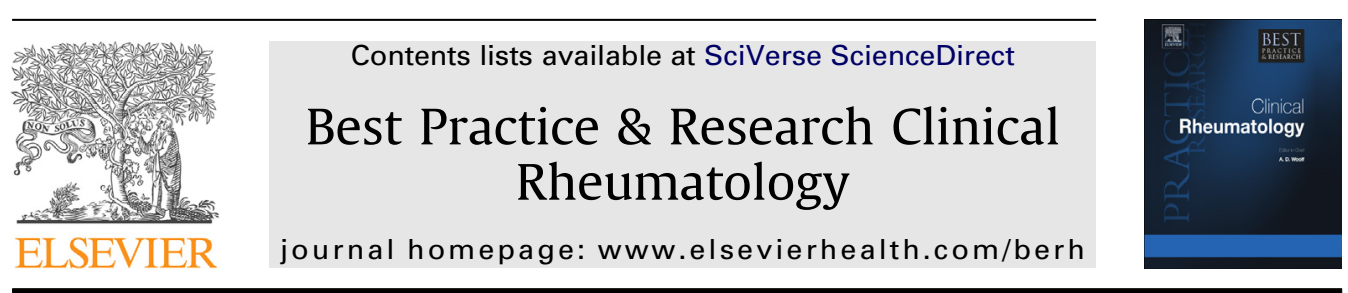

8

\title{
Becoming a musculoskeletal ultrasonographer
}

\author{
Annamaria Iagnocco ${ }^{\mathrm{a}, *}$, Esperanza Naredo ${ }^{\mathrm{b}}$, \\ Johannes W.J. Bijlsma ${ }^{c}$

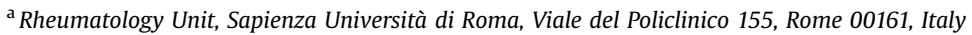 \\ ${ }^{\mathrm{b}}$ Department of Rheumatology, Hospital GU Gregorio Marañón, Madrid, Spain \\ ${ }^{\mathrm{c}}$ Department of Rheumatology \& Clinical Immunology, University Medical Center Utrecht, Utrecht, \\ The Netherlands
}

Keywords:

Competency education

Musculoskeletal ultrasonography

Training

\begin{abstract}
Over the last decade, increasing numbers of rheumatologists have incorporated musculoskeletal ultrasound (MSUS) as a valuable diagnostic tool into their clinical practice. Some countries have established training programmes for MSUS. The European League Against Rheumatism has developed education guidelines for the content and conductance of MSUS courses and it would be useful to standardise rheumatology MSUS training worldwide. A thorough knowledge of anatomy, US physics and technology, US scanning methods, US pattern of normal and pathological musculoskeletal tissues, definitions for US pathology, artefacts and pitfalls in both greyscale and Doppler modalities is necessary to perform efficient MSUS. MSUS training includes attending theoretical-practical and online courses, as well as studying textbooks and using digital video discs (DVDs). Having access to US equipment and performing supervised normal and pathological MSUS examinations for a training period are mandatory for consolidating MSUS learning. A proposal to accredit and certificate competence in MSUS is now being discussed.
\end{abstract}

(C) 2013 Elsevier Ltd. All rights reserved.

\section{Musculoskeletal ultrasound basis}

Ultrasonography (US) is an imaging technique based on the emission and reception of mechanical sound waves with a frequency greater than the hearing frequency range of the human ear (i.e., 20 kilohertz $(\mathrm{kHz})$ ) by piezoelectric crystals located inside the transducer or probe. Ultrasound wave

\footnotetext{
* Corresponding author.

E-mail address: Annamaria.Iagnocco@uniroma1.it (A. Iagnocco).
} 
frequencies of diagnostic US systems range from 3 megahertz (MHz) to $25 \mathrm{MHz}$. Ultrasound waves transmit differently through different media depending on their composition and are reflected at the interfaces between materials of different acoustic impedance. The reflection of ultrasound through the body tissues generates US images that consist of varying degrees of black-and-white images. The US technique includes greyscale imaging of anatomic structures and blood-flow detection by Doppler technique. Doppler US is based on the alteration of the frequency of a sound beam reflected back to the source when it encounters a moving object.

For the last 50 years, the medical applications of Doppler US have included diagnosis of abdominal, pelvic, obstetric, breast and cardiac pathologies. More recently, musculoskeletal ultrasonography (MSUS) has progressively become an established imaging technique for evaluating peri-articular and intra-articular structures involved in musculoskeletal conditions, including rheumatic diseases. The use of high-resolution transducers improves the visualisation of different anatomic structures and permits the assessment of a wide range of inflammatory and structural abnormalities. The use of appropriate probe frequencies is mandatory for the correct imaging of musculoskeletal structures and optimises the US detection of local pathology. At higher frequencies, the image resolution will be greater but the tissue penetration will be lower. Therefore, higher-frequency linear transducers (e.g., $10-18 \mathrm{MHz}$ ) should be used for superficial anatomic structures, such as the small joints of the hand and feet, whereas lower-frequency transducers (e.g., 5-10 MHz) can be used for deeper joints, such as the hip, ankle and shoulder. In addition, a correct machine setting markedly increases the sensitivity of the equipment in assessing a number of abnormalities, both by using greyscale and Doppler modalities.

US is a routinely available, dynamic, non-invasive and relatively inexpensive bedside imaging method that consent a multiplanar assessment of musculoskeletal system, with high patient acceptability. The technique facilitates the scanning of all peripheral joints as many times as required at the time of consultation. In addition, a multi-joint assessment can be performed during the same scanning session.

\section{Applications of MSUS in rheumatology}

Recent advances in technology have led to an extraordinary improvement in the quality and level of US equipment, with production of machines that consent a good visualisation of most superficial and deep musculoskeletal structures. Particularly, the great resolution of anatomic details at the level of superficial musculoskeletal structures offered by high-frequency transducers has promoted an increasing use of MSUS both in rheumatologic routine clinical practice and in research settings. In addition, the enhanced sensitivity for detecting low-velocity flow in small synovium, tendon and enthesis vessels achieved by recent colour Doppler and power Doppler techniques has led to the incorporation of Doppler US in rheumatology. The difficulties in detecting abnormalities within deep joints by physical examination facilitate the applications and use of US also in the assessment of pathology at the level of large joints.

MSUS allows an immediate correlation between imaging findings and clinical data, which improves diagnosis and management of patients with a range of rheumatic diseases from inflammatory arthritis, vasculitis or osteoarthritis to soft-tissue diseases [1-4]. MSUS has demonstrated more sensitivity than clinical evaluation in assessing joint inflammation [5-9]. There have been an increasing number of studies on the validity of Doppler US for evaluating inflammatory activity in arthritic joints [9-12]. Greyscale and Doppler US have an increasing role in therapy monitoring in inflammatory arthritis [1316] as well as in the evaluation of sub-clinical MSUS-detected synovitis, which can predict structural damage progression and disease relapse in patients with rheumatoid arthritis in clinical remission $[17,18]$. In addition ultrasound is a bedside tool for performing accurate and safe musculoskeletal injections [19].

\section{Education and training in MSUS}

Within the last years, increasing numbers of rheumatologists have incorporated MSUS into their clinical practice as a valuable imaging and research tool. Recent technological developments in the US systems resulting in the increasing utility of MSUS in rheumatology, the growing interest of 
rheumatologists in MSUS and the decreasing cost of sonographic machines have led to a great demand for appropriate education in this technique among rheumatologists worldwide. As MSUS is the most operator-dependant imaging modality [20,21] - mainly because of the intrinsic real-time nature of US image acquisition - appropriate training is highly important to ensure skilled and safe use of MSUS by rheumatologists.

In a survey of rheumatologists by the British Society for Rheumatology, the principal reason given for not performing MSUS was the lack of training in MSUS (75\% of respondents) [22]. More recently, a European survey responded by representative from 31 countries showed that in most countries few rheumatologists perform MSUS in clinical practice and that training in this technique varies widely from country to country, with low implementation of competency assessment [23]. A number of European countries run national training programmes for MSUS for rheumatologists. MSUS schools for rheumatologists, which can be supported by the national rheumatology societies or universities $[23,24]$, have been created in some countries. In addition, MSUS has been incorporated into fellowship programmes and postgraduate rheumatology training in some European countries [23]. However, there are probably still not enough rheumatologists and/or rheumatology centres able to provide MSUS training and most countries need to train new rheumatologist trainers in MSUS.

At present, many approaches are taken to teach MSUS. There is no standardised educational training programme in MSUS and no standard, accredited assessment of competency for rheumatologists performing MSUS. There are also not enough data on the outcome of training in MSUS, and the validity of current MSUS educational models has not been demonstrated. Thus, there is a need for a standardised curriculum, education guidelines and competency assessment in MSUS.

Fortunately, over the last years, a number of relevant papers on ultrasound education, curriculum and competency for rheumatologists have been published. These have contributed to the development of essential issues, MSUS training method and competency assessment in rheumatology [24-31]. More recently, training in ultrasound-guided injections for rheumatologists among countries has been tested and results demonstrated that the percentage of rheumatologists receiving training in the field is $<10 \%$ in over $70 \%$ of countries that participated in the survey [32].

\section{Knowledge and skills necessary to become a musculoskeletal ultrasonographer}

The knowledge and skills necessary to perform efficient and safe MSUS are shown in Box 1. A thorough knowledge of sectional anatomy, ultrasound physics and technology, joint sonographic scanning methods, the ultrasound pattern of normal and pathological musculoskeletal tissues, artefacts, diagnostic criteria and Doppler technique is all necessary for the correct performance of MSUS.

In 2001, the first guidelines for performing MSUS in rheumatology were published by the European League Against Rheumatism (EULAR) Working Group for Musculoskeletal Ultrasound [33]. These guidelines provided useful information on the technical basis for MSUS, equipment specifications, scanning methods and image acquisition along with the main pathological findings in each anatomical area.

\section{Box 1 Knowledge and skills necessary to perform musculoskeletal ultrasonography (MSUS)}

Sectional anatomy

US physics and technology

Joint US scanning method

US pattern of normal musculoskeletal tissues

Pathological MSUS findings

MSUS artefacts

MSUS diagnostic criteria

Doppler technique

Documentation and report of US findings 
Later on, Brown et al. produced an international interdisciplinary consensus on the specific indications, anatomic areas and knowledge and skills required by rheumatologists performing MSUS $[27,28]$.

A knowledge of the applications, indications and limitations of MSUS in rheumatology should be the starting point in MSUS education. A basic theoretical knowledge of ultrasound physics and technology is necessary to understand the diagnostic capabilities of this technique. After a short period of practical training, trainees can easily recognise the US pattern of the different musculoskeletal tissues. MSUS examination is the most difficult aspect of training and requires long practical sessions. The standardised and systematic scanning methods of peri-articular and intra-articular structures accessible by US - the shoulder, elbow, wrist and hand, hip, knee, ankle and foot - should be learnt in practical training after a solid understanding of joint sectional anatomy has been acquired. Multiplanar with longitudinal and transverse scans of musculoskeletal anatomic structures should be performed to acquire maximal morphological information. Dynamic examination provides functional assessment of musculoskeletal structures that facilitates diagnosis of abnormalities. The target joint structures for rheumatologists are tendons, bursae, synovial recesses, articular cartilages and bony cortex. Knowledge of US examination of ligaments, muscle and peripheral nerves enhances an overall US investigation of the musculoskeletal system. Holding the probe correctly and optimising the greyscale settings of the sonographic system are mandatory for an appropriate learning curve. MSUS artefacts and pitfalls should be learnt to avoid misdiagnosis of MSUS pathologies.

In most MSUS training programmes, 50-100 US scans of each anatomic area in normal subjects are strongly recommended before starting US diagnosis of musculoskeletal abnormalities. This educational approach enables trainees to acquire a skilled enough scanning technique and to acquire experience in normal anatomic variants before using MSUS as a diagnostic method.

Diagnostic applications of MSUS for rheumatologists are focussed in those pathological conditions in which MSUS has proven clinical value. Basic MSUS training for rheumatologists must comprise the ability to detect joint synovitis, bursitis, tenosynovitis, bone erosions and osteophytes, as well as the differentiation of fluid from synovial hypertrophy (Fig. 1). Moreover, trainees should be able to identify by US different tendon pathologies such as enthesopathy, tendon tear, tendinosis and tendon calcification. Articular cartilage lesions, peri- and intra-articular microcrystal deposits, myositis and joint and tendon ganglia and cysts should also be included in the MSUS curriculum for rheumatologist ultrasonographers.

In addition to greyscale MSUS, trainees should learn colour and power Doppler physics and technology, along with their application, indications and limitations in rheumatology. They need knowledge

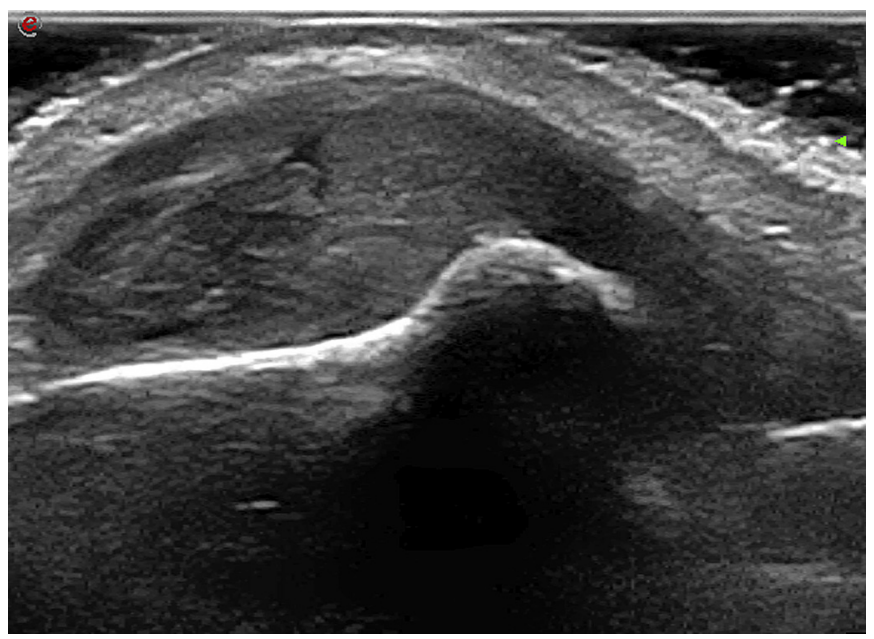

Fig. 1. Longitudinal ultrasound scan of a metacarpophalangeal joint. Greyscale image shows hypoechoic synovitis distending the joint capsule. 
of the colour and power Doppler settings and artefacts. Rheumatologist ultrasonographers should be trained in the detection and quantification of synovial, tenosynovial and entheseal inflammatory activity by Doppler US (Figs. 2 and 3). Additionally, at an advanced level, they could learn the role of US in vasculitis and be trained in the evaluation of target vessels and detection of vasculitis by US.

US-guided musculoskeletal interventional procedures, mainly peri-articular and articular injections, are a recommended skill for rheumatologists.

Pathological findings more common in other musculoskeletal specialities, such as peripheral nerve entrapment, ligament and fibrocartilage lesions, muscle injuries, soft-tissue masses and loose and foreign bodies, could be learnt optionally by rheumatologist ultrasonographers. MSUS in paediatric rheumatic diseases is a new and rapidly developing area for rheumatologists involved in this field. Finally, correct image documentation and appropriate reporting of ultrasound findings and diagnoses are important aspects of MSUS training.

The musculoskeletal pathologies included in the MSUS training curriculum for rheumatologists according to the recommendations for the content and conduct of EULAR MSUS courses are listed in Table 1 [31]. They are classified in three levels of training: basic, intermediate and advanced.

\section{Forms of MSUS training}

The principal forms of training in MSUS are shown in Box 2. Most rheumatologist ultrasonographers probably have learnt MSUS through a combination of them.

A number of rheumatologists learn from a mentor. Direct supervision by an expert is recognised as an appropriate training form. Unfortunately, only a limited number of mentors have enough time to provide training in MSUS. Attendance at theoretical and practical MSUS courses is strongly recommended, particularly in the early stages of training, but also at advanced level of training. In addition, the use of online ultrasound courses can be useful, particularly at the early phases of training and before starting basic courses, especially because they are able to provide basic information that can help in learning more rapidly during the attendance of residential courses. MSUS courses and workshops are offered by national societies of radiology and/or rheumatology and by universities in many countries [23,24]. Courses can be delivered locally or nationally, depending on the availability of trainers. They are conducted by radiologists, rheumatologists and other specialist ultrasonographers.

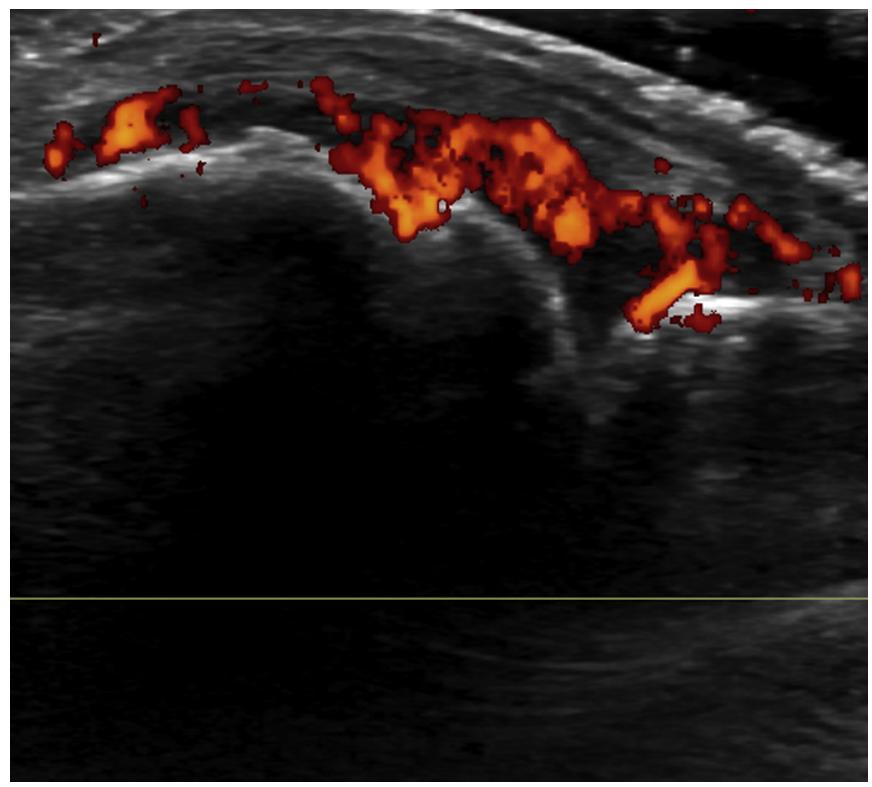

Fig. 2. Longitudinal ultrasound scan of a metacarpophalangeal joint which shows synovitis with synovial Doppler signal. 


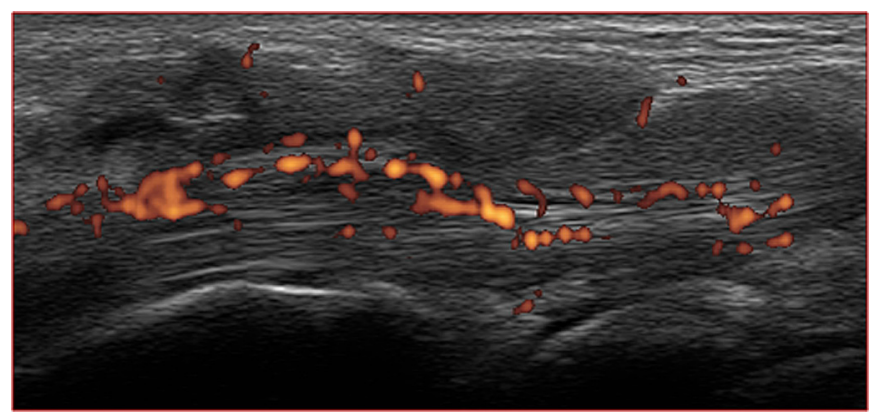

Fig. 3. Longitudinal ultrasound scan of the flexor tendons (II finger) showing tenosynovitis with synovial and intra-tendinous Doppler signal.

Table 1

Pathology content for basic, intermediate and advanced MSUS training levels.

\begin{tabular}{lll}
\hline Basic level & Intermediate level & Advanced level \\
\hline Joint synovitis & Tendon calcification & Peripheral nerve entrapment and lesions \\
Joint effusion & Enthesopathy & Ligament lesions \\
Synovial hypertrophy & Tendinosis & Fibrocartilage lesions \\
Bursitis & Paratenonitis & Myopathy \\
Tenosynovitis & Tendon subluxation/luxation & Myositis \\
& Intrasubstance tendon lesions & Muscle injury \\
& Tendon impingement & Soft-tissue masses \\
& Complete tendon tear & Loose bodies \\
& Partial tendon tear & Foreign bodies \\
\hline
\end{tabular}

Osteophytes ganglia and cysts. Articular cartilage lesions. Peri- and intra-articular microcrystal deposit Ligament, muscle, cartilage, fibrocartilage and synovial calcification. MSUS, musculoskeletal ultrasonography.

The EULAR has supported 19 courses on MSUS since 1998. There have been introductory, intermediate and/or advanced MSUS courses in different European countries under the auspices of the EULAR Standing Committee on Education and Training. European rheumatologists highly experienced in MSUS have comprised the faculty of these courses. Many of them chair and organise MSUS training for rheumatologists in their own countries. There has been a full and enthusiastic attendance at these courses.

In 2007, the rheumatologists (from 11 European countries) who make up the faculty giving these courses developed educational guidelines for the content and conduct of EULAR ultrasound courses; these are recommended for national and local MSUS training programmes. They might also be useful for standardising rheumatology MSUS training worldwide [31].

The group consensus on EULAR guidelines and curriculum proposes a three-level education model: basic, intermediate and advanced. Basic courses focus on examination technique and include some basic pathology. Intermediate courses focus on a wide spectrum of rheumatological pathology and the basic use of Doppler and musculoskeletal US-guided injections. Advanced courses focus on advanced

\section{Box 2 Forms of musculoskeletal ultrasonography (MSUS) training}

$\square$ Mentor

$\square$ Theoretical and practical courses

$\square$ Formal/informal training from radiologists/rheumatologists

$\square$ E-learning

$\square$ Learning sonoanatomy in specimens

$\square$ Self-teaching 
Doppler, current MSUS research in rheumatology, new technological developments, uncommon pathological findings in rheumatology, pathological findings in other specialities (such as nerve, ligament, muscle lesions and sport-related lesions) and MSUS methodology. The recommended course duration is $20 \mathrm{~h}$. There should be a maximum of six participants per teacher and $50-60 \%$ of the total time should be spent in practical sessions.

Training after courses, under expert supervision as much as possible, by performing normal scans and diagnostic examinations is essential to consolidate the knowledge and skills provided during the courses and should be a compulsory requisite before trainees perform MSUS independently.

Formal or informal training from a colleague expert in MSUS, predominantly a rheumatologist or radiologist, has been widely used among rheumatologist ultrasonographers to achieve MSUS competency [29].

Among the various EULAR initiatives, in 2012 a EULAR online MSUS introductory course was also started with the aim of providing rheumatologists a basic theoretical knowledge on MSUS and later on will continue a training programme by attending future EULAR courses and/or MSUS courses with EULAR endorsement (For actual info visit: www.eular.org/education).

The course, which represents an electronic form of continuous medical education in rheumatology, is composed of seven modules that run entirely through the web. It gives theoretical basic skills on MSUS at different joint sites, in healthy subjects and in rheumatic diseases, covering different aspects of MSUS in rheumatology.

In addition to the EULAR online course, other online courses are available on the web. E-learning can be a complementary form of training in MSUS. The objective of e-learning is to provide training to a wide audience. Clearly, a website cannot substitute for continuous practice. However, whereas the image-acquisition process in US training requires local expert supervision, the interpretation process can be assisted by experts via the Internet. E-learning allows continuous educational interaction between trainees and tutors [30].

Finally, MSUS training in sonoanatomy by direct visualisation of anatomic structures in cadaver specimens can be an additional form of improving understanding of anatomy and scanning skills.

Until now, many rheumatologists and radiologists have been self-taught, by performing a large number of normal and pathological scans, studying books or other imaging educational tools and comparing their results with other imaging techniques, such as magnetic resonance imaging (MRI). Self-taught ultrasonographers have to be highly motivated and have a considerable amount of free time. Although possible, this is not the best way to achieve training and competency in MSUS.

Following MSUS training, regular continuing medical education is necessary to ensure that the knowledge and skills in MSUS are maintained when ultrasonographers practice independently. Teacher-training courses are also recommended to ensure that trainers maintain their teaching capability. For those who want to be involved in (local) US courses as a trainer a 'teach the teachers' course has been developed, presently preceding the annual EULAR US courses.

\section{MSUS educational tools}

Different educational tools that can be used to learn MSUS are listed in Box 3. There are a number of relevant textbooks [34-41] and DVDs (e.g., Musculoskeletal ultrasound, a beginner's guide to normal peripheral joint anatomy. Kane D, Balint P, Sturrock R. Arthritis Research Campaign (arc), www.arc.org.

\section{Box 3 Musculoskeletal ultrasonography (MSUS) educational tools}

Atlas on sectional musculoskeletal anatomy

$\square$ Textbooks on MSUS

$\square$ Published articles on MSUS (review, research and update papers)

$\square$ DVDs on MSUS (musculoskeletal sonoanatomy, scanning technique, and/or sonopathology)

$\square$ Websites 
uk) on different aspects of MSUS education such as sonoanatomy, scanning technique and pathology (Fig. 4). There are also various free websites that include sectional anatomy, US images of normal musculoskeletal system and a wide spectrum of pathologies along with educational texts (e.g., http:// www.irheum.eu; http://www.eular.org; http://www.aium.org; http://www.med.umich.edu/rad/ muscskel/mskus; http://www.efsumb.org; and http://www.essr.org). These educational tools facilitate practical MSUS learning. An in-depth knowledge of sectional musculoskeletal anatomy should be acquired before starting any training in MSUS.

\section{Technical equipment}

The principal tool for becoming an MSUS ultrasonographer is US equipment. Having access to an appropriate US machine is essential in learning and developing MSUS skills. Ultrasound equipment for musculoskeletal imaging needs to have high-frequency broadband (7.5-18 MHz) linear transducers for demonstrating superficial structures including tendons, ligaments and small joints, colour Doppler and power Doppler techniques and sufficient resolution for imaging the anatomic details that are important to rheumatologists. In addition, lower-frequency probes should also be available, in order to consent the assessment of large joints and deep musculoskeletal structures. Currently, portable highquality US machines are available.

The price of US equipment ranges approximately from 20,000 to 130,000 euros, according to the quality and the availability of additional imaging procedures (e.g., wide-band Doppler imaging, spatial
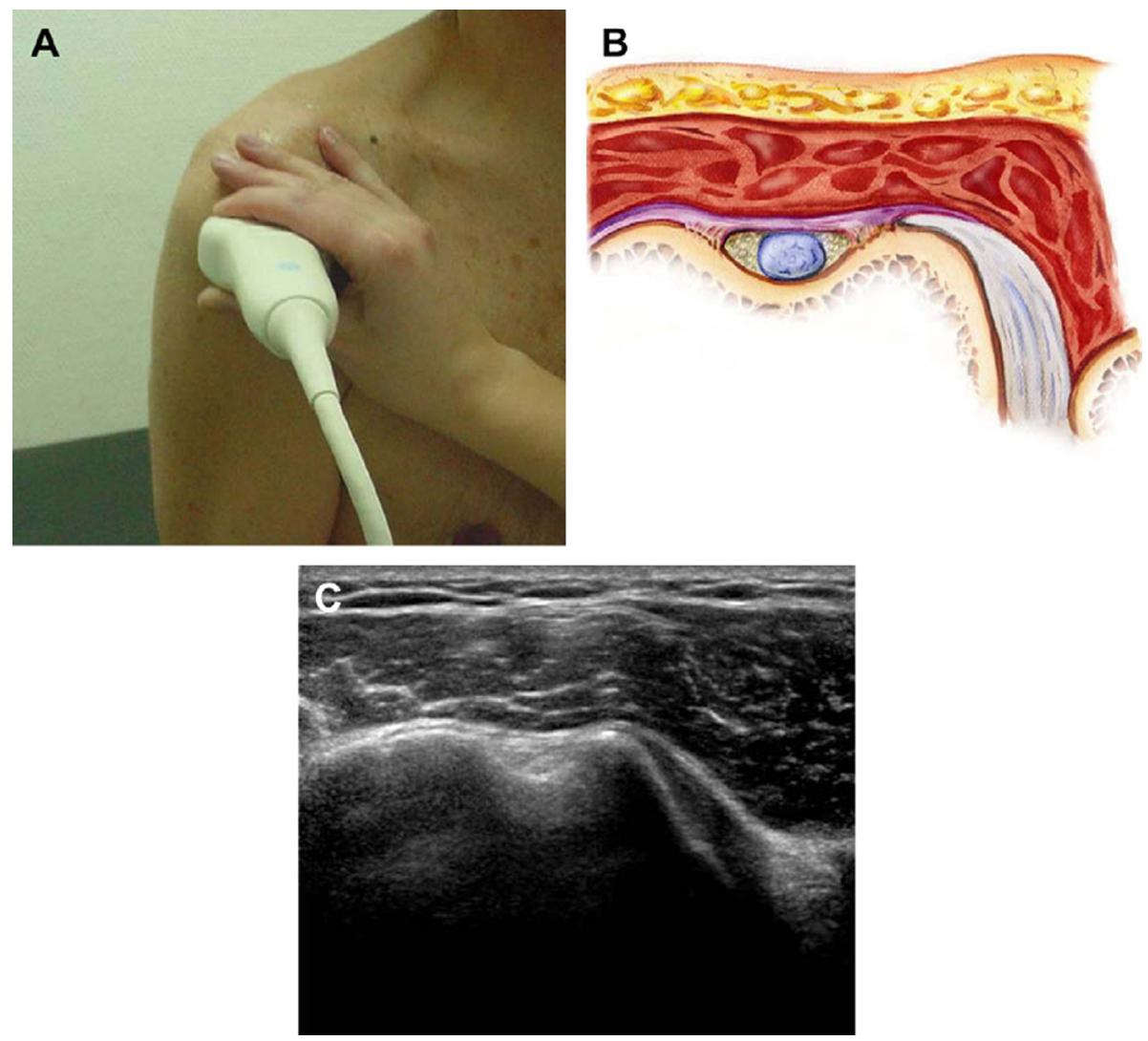

Fig. 4. Example of a basic book or DVD on MSUS that shows patient position and probe placement (A), sonoanatomy (B) and normal sonographic imaging $(C)$ (transverse scan of the biceps tendon at the bicipital groove in these images). 
compound imaging, extended field-of-view imaging, steering-based imaging and three-dimensional (3D) imaging). Frequently, the funding for equipment represents a problem. Funders and health authorities need to be aware and become convinced of the impact of MSUS in clinical practice, its costeffectiveness and its benefits to patients. Research grants can represent alternative means of funding US equipment.

\section{Recognition and certification as a rheumatologist ultrasonographer}

US in rheumatic diseases has now become an important part of daily practice in many situations. It is therefore the task of the rheumatologic community to make sure that the quality of these examinations and interventions meets the required standards. EULAR, the umbrella organisation of the scientific rheumatology societies of all European countries (as well as patients' organisations and allied healthcare organisations), has always been very supportive of education in this field. EULAR has also stimulated research to find the best place for US applications. EULAR now considers it appropriate to develop a process that will lead ultimately to some form of official recognition of MSUS competency by rheumatologists. In co-operation with EULAR, a structure is being developed to award European accreditation. The training programme laid out in the three different levels of US courses may form the basis of this process. The following set-up is proposed:

* Only those who can use a US machine on a regular basis are able to start training.

* The training starts with the basic US course; thereafter the trainee performs at least 100 US examinations in that area (see Table 1 ) in the following year.

* In the second year, the trainee follows the intermediate US course and, in the following year, will perform at least 200 US examinations in the areas of the basic and intermediate courses (see Table 1).

* In the third year, the trainee will follow the advanced course and after that there will be a practical examination. Trainees who successfully pass this examination will receive a certificate of having shown enough theoretical and practical knowledge to be awarded the EULAR certification of 'ultrasonographer in rheumatology'.

How should this examination be organised? The following proposal has been made, but the final process still needs to be defined. It is suggested that, for the examination, a large room is set up with 12 US stations, all staffed by two experienced and qualified teachers and by a patient with a certain condition. The candidates will pass all these stations and need to show to the teachers that they can perform the right US, make the right diagnosis and suggest the right interventions. The teachers can ask the candidates additional questions on the specific problem at hand, or more general questions. The candidates need to perform well in at least 10 of these 12 stations, according to the judgement of the two teachers present at each station. Such practical examinations have proved feasible and effective in many situations, for example, for the European ophthalmology examinations. The first group of about 30 qualified teachers will be appointed after proposals by the European MSUS community, which has been responsible for these courses for over 10 years. Further teachers can qualify in the future when they have successfully passed the examination described above, have a practical experience of at least 3 years and have successfully attended the 'teach the teachers' course. EULAR has become a trademark for high-quality education and patient care in the field of rheumatology and plays an important role in the development of these activities.

At present, procedures are under development and, hopefully, this will lead to high-quality, certified rheumatologist ultrasonographers.

Recently, a core group of European rheumatologists highly involved in national and international MSUS education and training has been formed under the umbrella of the European Federation of Societies for Ultrasound in Medicine and Biology (EFSUMB). This group has worked in adapting the theoretical and practical training requirements and appropriate competency assessment for rheumatologists to the three-level competency assessment system published by EFSUMB in 2006 (www.efsumb.org/guidelines). 
Practice points: How to become a musculoskeletal ultrasonographer

- Need access to US equipment.

- Do independent study using books, DVDs, websites and papers on MSUS.

- Attend MSUS courses.

- Practise normal and pathological MSUS under expert supervision.

- Participate in MSUS research.

\section{Summary points and research agenda}

Key points for becoming a musculoskeletal ultrasonographer include having access to an appropriate ultrasound machine, using educational tools, regularly attending MSUS courses, practising normal and pathological MSUS under expert supervision and, if possible, participating in research studies on MSUS. At present, most European countries need to train new rheumatologist trainers in MSUS. In addition, there is a need for a standardised curriculum and education guidelines to efficiently train rheumatologist ultrasonographers. Competency assessment should be developed to ensure that adequate knowledge and skills in MSUS are achieved and maintained.

\section{References}

[1] Grassi W, Cervini C. Ultrasonography in rheumatology: an evolving technique. Annals of the Rheumatic Diseases 1998;57: 268-70.

[2] Wakefield RJ, Brown AK, O'Connor PJ, Karim Z, Grainger A, Emery P. Musculoskeletal ultrasonography: what is it and should training be compulsory for rheumatologists? Rheumatology 2004;43:821-2.

[3] Kane D, Grassi W, Sturrock R, Balint PV. Musculoskeletal ultrasound-a state of the art review in rheumatology. Part 2: clinical indications for musculoskeletal ultrasound in rheumatology. Rheumatology 2004;43:829-38.

[4] Schmidt WA, Seifert A, Gromnica-Ihle E, Krause A, Natusch A. Ultrasound of proximal upper extremity arteries to increase the diagnostic yield in large-vessel giant cell arteritis. Rheumatology 2008;47:96-101.

[5] Kane D, Balint PV, Sturrock RD. Ultrasonography is superior to clinical examination in the detection and localization of knee joint effusion in rheumatoid arthritis. Journal of Rheumatology 2003;30:966-71.

*[6] Wakefield RJ, Green MJ, Marzo-Ortega H, Conaghan PG, Gibbon WW, McGonagle D, et al. Should oligoarthritis be reclassified? - ultrasound reveals a high prevalence of subclinical disease. Annals of the Rheumatic Diseases 2004;63: 382-5.

[7] Karim Z, Wakefield RJ, Quinn M, Conaghan PG, Brown AK, Veale DJ, et al. Validation and reproducibility of ultrasonography in the detection of synovitis in the knee: a comparison with arthroscopy and clinical examination. Arthritis \& Rheumatism 2004;50:387-94.

[8] Szkudlarek M, Narvestad E, Klarlund M, Court-Payen M, Thomsen HS, Østergaard M. Ultrasonography of the metatarsophalangeal joints in rheumatoid arthritis: comparison with magnetic resonance imaging, conventional radiography, and clinical examination. Arthritis \& Rheumatism 2004;50:2103-12.

*[9] Naredo E, Bonilla G, Gamero F, Uson J, Carmona L, Laffon A. Assessment of inflammatory activity in rheumatoid arthritis: a comparative study of clinical evaluation with grey scale and power Doppler ultrasonography. Annals of the Rheumatic Diseases 2005;64:375-81.

[10] Walther M, Harms H, Krenn V, Radke S, Faehndrich TP, Gohlke F. Correlation of power Doppler sonography with vascularity of the synovial tissue of the knee joint in patients with osteoarthritis and rheumatoid arthritis. Arthritis \& Rheumatism 2001;44:331-8.

[11] Szkudlarek M, Court-Payen M, Strandberg C, Klarlund M, Klausen T, Ostergaard M. Power Doppler ultrasonography for assessment of synovitis in the metacarpophalangeal joints of patients with rheumatoid arthritis: a comparison with dynamic magnetic resonance imaging. Arthritis \& Rheumatism 2001;44:2018-23.

[12] Terslev L, Torp-Pedersen S, Savnik A, von der Recke P, Qvistgaard E, Danneskiold-Samsøe B, et al. Doppler ultrasound and magnetic resonance imaging of synovial inflammation of the hand in rheumatoid arthritis: a comparative study. Arthritis \& Rheumatism 2003;48:2434-41.

*[13] Filippucci E, Iagnocco A, Salaffi F, Cerioni A, Valesini G, Grassi W. Power Doppler sonography monitoring of synovial perfusion at the wrist joints in patients with rheumatoid arthritis treated with adalimumab. Annals of the Rheumatic Diseases 2006;65:1433-7.

*[14] Naredo E, Collado P, Cruz A, Palop MJ, Cabero F, Richi P, et al. Longitudinal power Doppler ultrasonographic assessment of joint inflammatory activity in early rheumatoid arthritis: predictive value in disease activity and radiological progression. Arthritis \& Rheumatism 2007; $15: 116-24$.

*[15] Iagnocco A, Filippucci E, Perella C, Ceccarelli F, Cassarà E, Alessandri C, et al. Clinical and ultrasonographic monitoring of response to adalimumab treatment in rheumatoid arthritis. Journal of Rheumatology 2008;35:35-40. 
*[16] Naredo E, Rodríguez M, Campos C, Rodríguez-Heredia JM, Medina JA, Giner E, et al. Validity, reproducibility and responsiveness of a 12-joint simplified power Doppler ultrasonographic assessment of joint inflammation in rheumatoid arthritis. Arthritis \& Rheumatism 2008;59:512-22.

*[17] Brown AK, Conaghan PG, Karim Z, Quinn MA, Ikeda K, Peterfy CG, et al. An explanation for the apparent dissociation between clinical remission and continued structural deterioration in rheumatoid arthritis. Arthritis \& Rheumatism 2008; 58:2958-67.

[18] Foltz V, Gandjbakhch F, Etchepare F, Rosenberg C, Tanguy ML, Rozenberg S, et al. Power Doppler ultrasound, but not lowfield magnetic resonance imaging, predicts relapse and radiographic disease progression in rheumatoid arthritis patients with low levels of disease activity. Arthritis \& Rheumatism 2012;64:67-76.

[19] Koski JM. Ultrasound-guided injections in rheumatology. Journal of Rheumatology 2000;27:2131-8.

[20] Scheel AK, Schmidt WA, Hermann KG, Bruyn GA, D'Agostino MA, Grassi W, et al. Interobserver reliability of rheumatologists performing musculoskeletal ultrasonography: results from a EULAR "train the trainers" course. Annals of the Rheumatic Diseases 2005;64:1043-9.

[21] Naredo E, Möller I, Moragues C, de Agustín JJ, Scheel AK, Grassi W, et al. Interobserver reliability in musculoskeletal ultrasonography: results from a "teach the teachers" rheumatologist course. Annals of the Rheumatic Diseases 2006;65: 14-9.

[22] Cunnington J, Platt P, Raftery G, Kane D. Attitudes of United Kingdom rheumatologists to musculoskeletal ultrasound practice and training. Annals of the Rheumatic Diseases 2007;66:1381-3.

*[23] Naredo E, D’Agostino MA, Conaghan PG, Backhaus M, Balint P, Bruyn GA, et al. Current state of musculoskeletal ultrasound training and implementation in Europe: results of a survey of experts and scientific societies. Rheumatology 2010; 49:2438-43.

[24] Uson J, Naredo E. Snap-shot of the ultrasound school of the Spanish Society of Rheumatology. Reumatismo 2005;57:1-4.

*[25] D’Agostino MA, Maillefert JF, Said-Nahal R, Breban M, Ravaud P, Dougados M. Detection of small joint synovitis by ultrasonography: the learning curve of rheumatologists. Annals of the Rheumatic Diseases 2004;63:1284-7.

[26] Brown AK, O'Connor PJ, Wakefield RJ, Roberts TE, Karim Z, Emery P. Practice, training, and assessment among experts performing musculoskeletal ultrasonography: towards the development of an international consensus of educational standards for ultrasonography for rheumatologists. Arthritis \& Rheumatism 2004;51:1018-22.

[27] Brown AK, O'connor PJ, Roberts TE, Wakefield RJ, Karim Z, Emery P. Recommendations for musculoskeletal ultrasonography by rheumatologists: setting global standards for best practice by expert consensus. Arthritis \& Rheumatism 2005;53:83-92.

[28] Brown AK, O'Connor PJ, Roberts TE, Wakefield RJ, Karim Z, Emery P. Ultrasonography for rheumatologists: the development of specific competency based educational outcomes. Annals of the Rheumatic Diseases 2006;65:629-36.

[29] Taggart A, Filippucci E, Wright G, Bell A, Cairns A, Meenagh G, et al. Musculoskeletal ultrasound training in rheumatology: the Belfast experience. Rheumatology 2006;45:102-5.

[30] Filippucci E, Meenagh G, Ciapetti A, lagnocco A, Taggart A, Grassi W. E-learning in ultrasonography: a web based approach. Annals of the Rheumatic Diseases 2007;66:962-5.

[31] Naredo E, Bijlsma JW, Conaghan PG, Acebes C, Balint P, Berner-Hammer H, et al. Recommendations for the content and conduct of EULAR musculoskeletal ultrasound courses. Annals of the Rheumatic Diseases 2008;67:1017-22. http://dx.doi. org/10.1136/ard.2007.082560. originally published online 5 Oct 2007; Annals of the Rheumatic Diseases 2007.

[32] Mandl P, Naredo E, Conaghan PG, D'Agostino MA, Wakefield RJ, Bachta A, et al. Practice of ultrasound-guided arthrocentesis and joint injection, including training and implementation, in Europe: results of a survey of experts and scientific societies. Rheumatology 2012;51:184-90.

*[33] Backhaus M, Burmester GR, Gerber T, Grassi W, Machold KP, Swen WA, et al. Guidelines for musculoskeletal ultrasound in rheumatology. Annals of the Rheumatic Diseases 2001;60:641-9.

[34] van Holsbeeck MT, Introcaso JH. Musculoskeletal ultrasound. 2nd ed. St Louis: Mosby; 2001.

[35] Marcelis S, Daenen B, Ferrara MA, Dondelinger RF, editors. Peripheral musculoskeletal ultrasound atlas. Stuttgard: Thieme; 1996.

[36] Bradley M, O’Donnell P. Atlas of musculoskeletal ultrasound anatomy. New York: Cambridge University Press; 2004.

[37] McNally EG. In: Houston M, editor. Practical musculoskeletal ultrasound. Philadelphia: Elsevier; 2005.

[38] Bianchi S, Martinoli C, Baert AL, Knauth M, Sartor K, editors. Ultrasound of the musculoskeletal system. Berlin: Springer; 2007.

[39] Banderali S, Bartolini B, Cesarano E. In: Martino F, Silvestri E, Grassi W, Garlaschi G, editors. Musculoskeletal sonography. Technique, anatomy, semeiotics and pathological findings in rheumatic diseases. Milano: Springer; 2007.

[40] Bruyn GAW, Schmidt W. Introductory guide to musculoskeletal ultrasound for the rheumatologist. Houten, NL: Bohn Stafleu van Loghum; 2006.

[41] Naredo E, Uson J, Acebes C. In: Naredo E, editor. Joint ultrasonography. Sonoanatomy and examination technique. Barcelona: Euromedice, Ediciones Médicas SL; 2007. 